\title{
Research on Defect Detection Technology of Composite Insulator Sheath
}

\author{
Wenbo $\mathrm{Li}^{1 *}$, Dun $\mathrm{Mao}^{2}$, Yue Jiang ${ }^{3}$, Lanlan $\mathrm{Liu}^{2}$, Jiran Zhu ${ }^{1}$, Tao Wan ${ }^{1}$, Fanglin Zha ${ }^{1}$, Yafeng Chao ${ }^{1}$ \\ ${ }^{1}$ State grid hunan electric power company limited research institute, Changsha, Hunan, 410007, China \\ ${ }^{2}$ State Grid Hunan Transmission Maintenance, Changsha, Hunan, 410000, China \\ ${ }^{3}$ State grid hunan electric power company limited, Changsha, Hunan, 410000, China
}

\begin{abstract}
The composite insulator sheath plays an important role in protecting the core rod and insulation. The thickness of the sheath must meet the corresponding standard requirements. During the insulator manufacturing process, the core rod decentration leads to uneven thickness of the sheath, which is easy to cause the risk of composite insulators premature failure during operation. The phased array detection technology can accurately detect the thickness of the sheath, so that defects such as uneven thickness of the sheath (decentration of the core rod) can be detected, and the thickness of the sheath at the end of the composite insulator can also be effectively detected. DR detection technology can effectively identify the interface between the mandrel and the sheath, but there is a risk of the sheath thickness distortion due to inappropriate transillumination parameters.
\end{abstract}

\section{Introduction}

Composite insulators are widely used in high-voltage power transmission systems because of their light weight, high tensile strength, and strong pollution resistance. Due to the influence of manufacturing process, service life and operating environment, the core rod brittle fracture caused by internal defects of composite insulators often causes transmission line accidents. To ensure the safe operation of transmission lines, how to effectively detect the internal defects of composite insulators has become an issue of great concern to the power sector [1,2].

In the manufacturing process of composite insulators, due to the long core rod, the middle section of the core rod will be bounced during the sheath molding process, and the core rod is easy to be eccentric, which leads to the defect of uneven sheath thickness locally. The sheath can protect the mandril and insulate the composite insulator. If the thickness of the sheath is uneven, the thickness of the local sheath is insufficient, and the insulator will be prone to early damage to the core rod during service $[3,4]$.

The basic idea of ultrasonic phased array technology comes from radar electromagnetic wave phased array technology. Phased array radar is composed of many radiating elements arranged in an array. By controlling the amplitude and phase of each element in the array antenna, the radiation direction of electromagnetic waves can be adjusted to synthesize flexible and fast focused scanning radar beams within a certain space. The ultrasonic phased array transducer is composed of a plurality of independent piezoelectric wafers. According to certain rules and timing sequence, each chip unit is excited by the electronic systems controlling to adjust the position and direction of the focus.

DR inspection radiation digital imaging inspection (DR) Radiation digital imaging inspection principle Radiation transilluminates the inspected workpiece, the attenuated ray photons are received by the digital detector, and after a series of conversions, they are transformed into digital signals. After amplification and A/D conversion, the digital signal is processed by computer and output in the form of digital image on the display. Compared with film, digital imaging has the characteristics of high sensitivity, low radiation dose required, short exposure, high detection efficiency, and avoiding the film washing in the darkroom $[5,6]$. In this paper, ultrasonic phased array detection technology and DR detection technology will be studied in the detection of potential defects of composite insulators.

\section{Test methods}

Ultrasonic phased array equipment adopts Phascan of Guangzhou Doppler Electronic Technologies Co., Ltd., and adopts the developed 7.5S32-0.5x10-V14(R25)-U110-2.5 self-focusing curved surface probe to conduct sheath thickness detection. DR detection uses General Electric Company's DR detection technology.

\section{Results and analysis}

\subsection{Ultrasonic phased array detection}

Due to the obvious difference in acoustic impedance

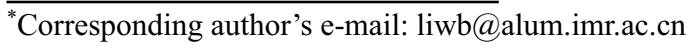


between the sheath and the core rod and fittings, the reflection is obvious, so an obvious reflected wave is formed at the interface between the sheath and the core rod to detect the thickness of the sheath. For samples with uneven thickness of the insulator sheath, phased array

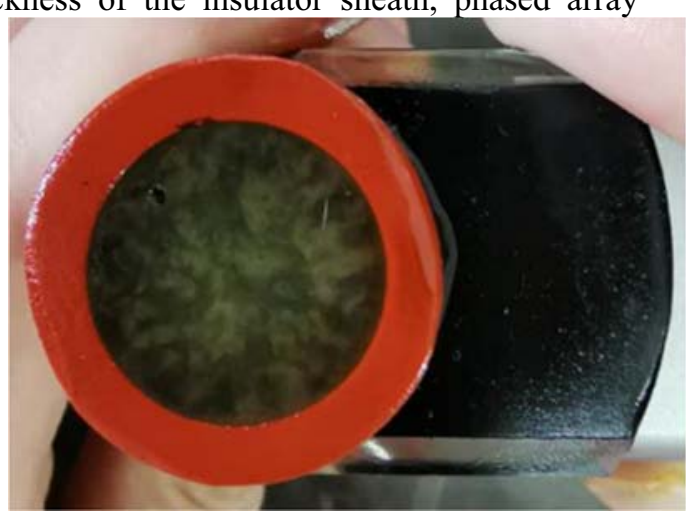

probes are used to detect the thickness. Circumferential inspection can find that the thickness of the composite insulator sheath is uneven, and the thickness of the sheath varies from 3.0 to $3.9 \mathrm{~mm}$. This detection method can be used for effective detection.
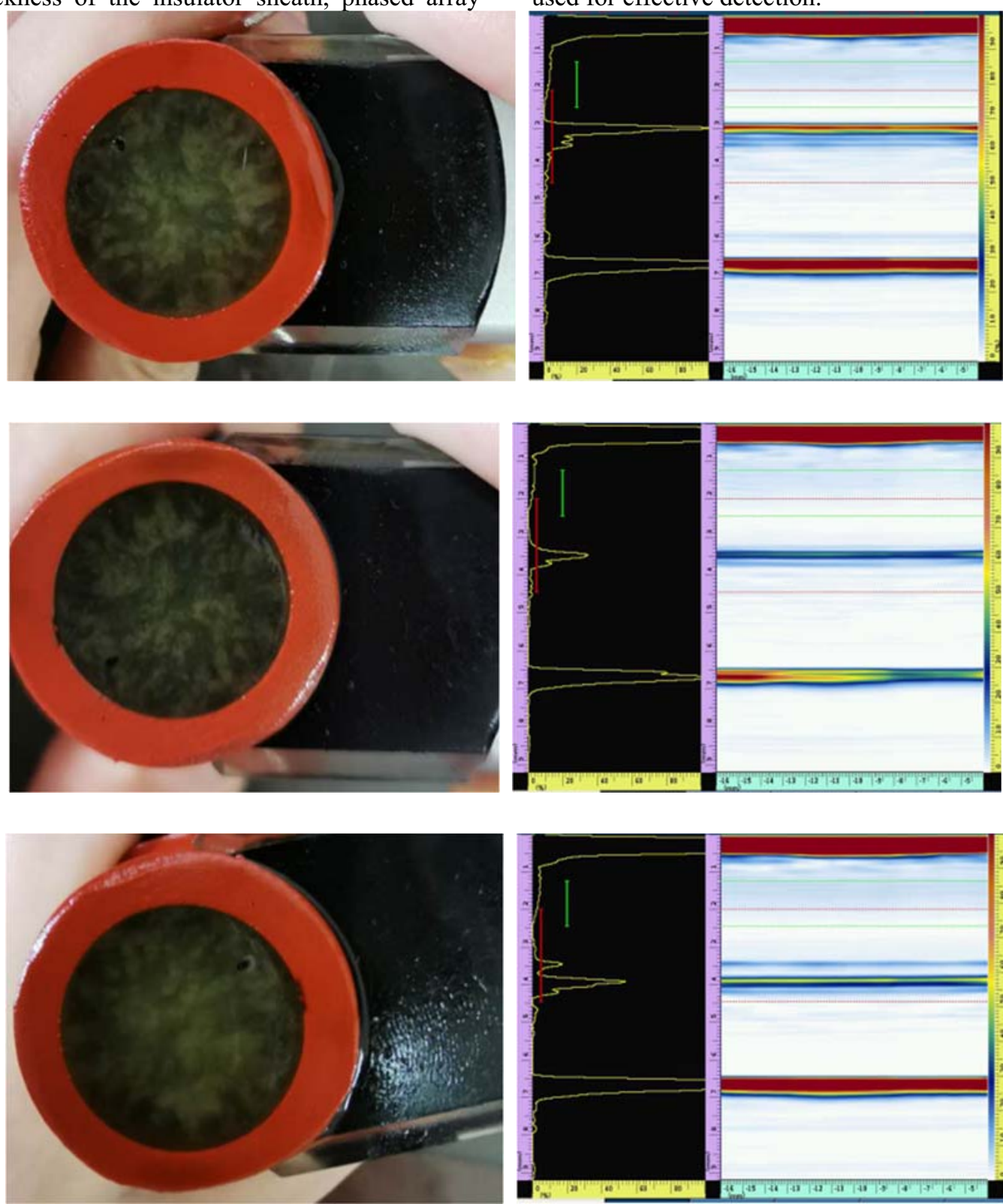

Figure 1 The actual detection of composite insulators with uneven sheath thickness

The thickness of the end sheath of the composite insulator is tested, and the self-focusing curved array probe and matching wedge are used to test the thickness of the $10 \mathrm{kV}$ composite insulator end sheath and the $35 \mathrm{kV}$ composite insulator end sheath, and the end sheath thickness is $2.4 \mathrm{~mm}$; for the $35 \mathrm{kV}$ composite insulator workpiece, the end sheath thickness is $8.4 \mathrm{~mm}$. The sheath thickness of $35 \mathrm{kV}$ composite insulator workpiece's straight rod section was tested, and the sheath thickness was $4.0 \mathrm{~mm}$. 

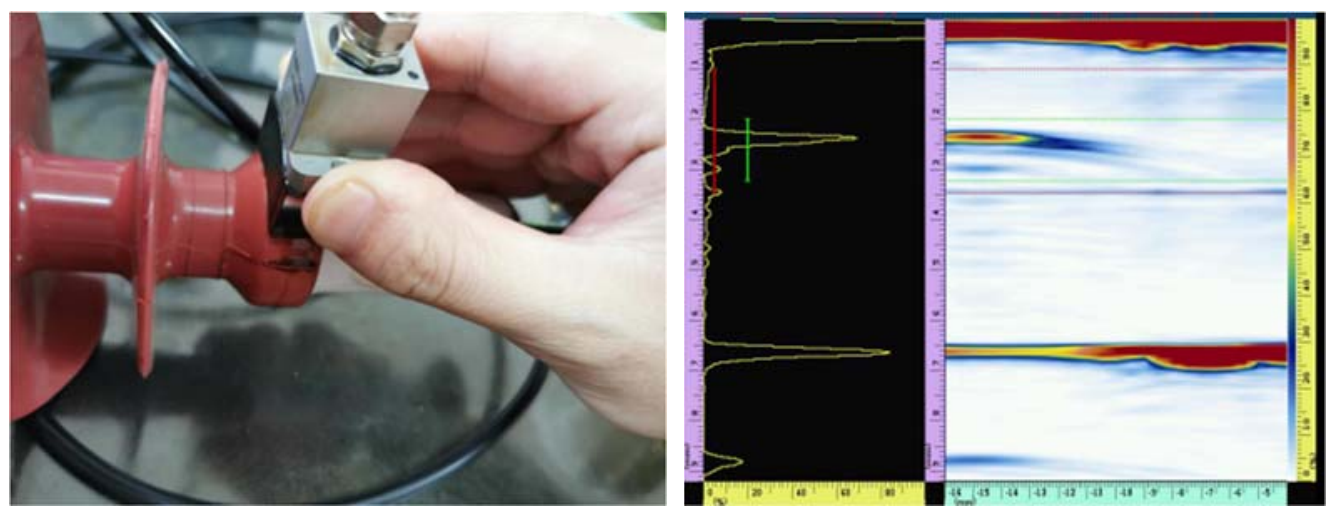

Figure $210 \mathrm{kV}$ composite insulation end sheath thickness detection
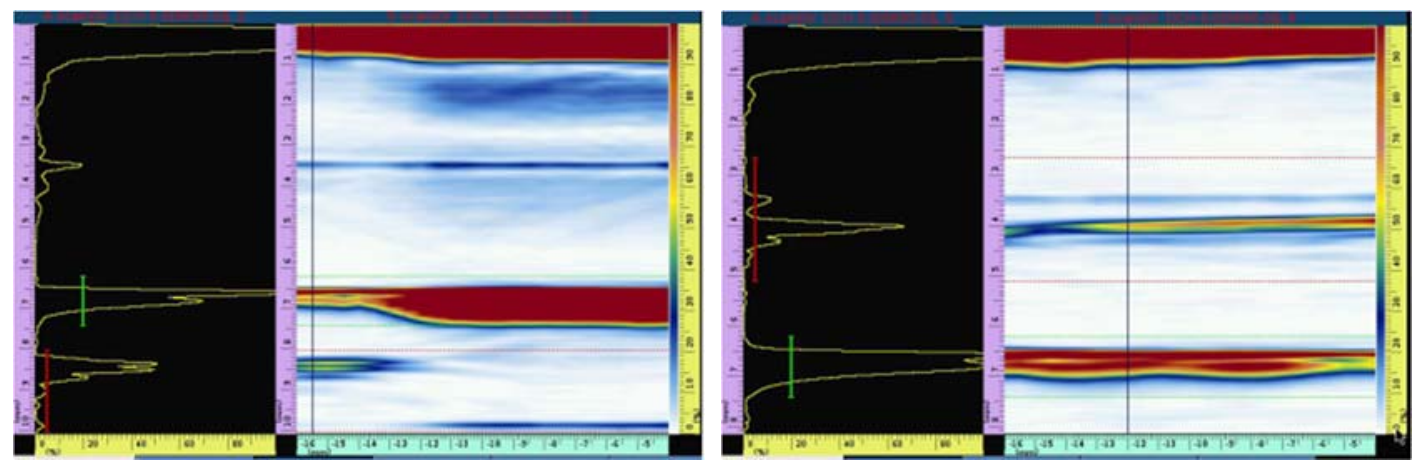

Figure $335 \mathrm{kV}$ composite insulator end thickness and core rod thickness detection

\subsection{DR detection}

Since the transmission intensity of the core rod and the sheath, and the sheath and the fittings are quite different, it can be clearly distinguished in imaging. Using appropriate transillumination voltage and current, the interface between the core rod and the sheath can be clearly displayed. It is possible to detect the thickness of the composite insulator sheath through DR detection technology $[7,8]$. However, the problem of using DR detection technology to detect the sheath thickness of composite insulators lies in that, when the reflected voltage and current are not selected properly, overexposure of the sheath surface is likely to occur, resulting in the sheath imaging thickness being smaller than the real thickness and difficult to be found in the image. Taking the same batch of $35 \mathrm{kV}$ composite insulators as an example, when the transillumination voltage increases from $50 \mathrm{kV}$ to $55 \mathrm{kV}$, the imaging thickness of the sheath decreases to about half of the actual value, as shown in Figures 4 and 5. Detecting the thickness of the sheath at the end of the composite insulator, DR imaging can clearly show the sealing structure of the end of the workpiece, and the interface between the fitting and the sheath is clear, as shown in Figure 6. Of course, there is also the problem of inaccurate sheath imaging thickness caused by overexposure. This is shown more clearly in Figure 7. The same three $110 \mathrm{kV}$ composite insulator samples have obvious sheath thickness under different transillumination voltages and currents. Therefore, it is a better choice to use phased array detection technology to detect the thickness of the composite insulator sheath.

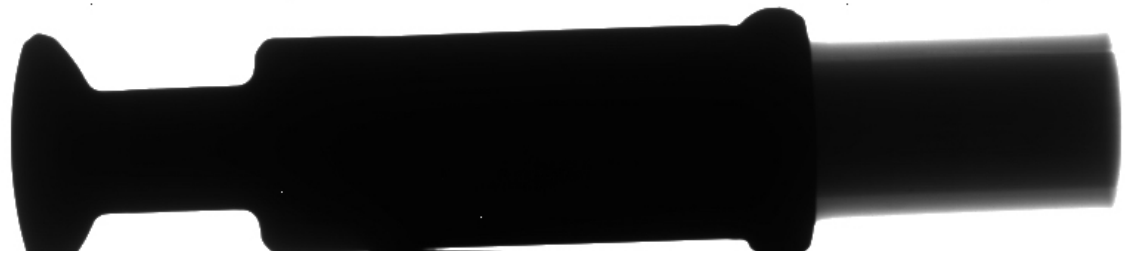

Figure $4 \mathrm{DR}$ detection at the end of composite insulator $(50 \mathrm{kV}, 3 \mathrm{~mA})$

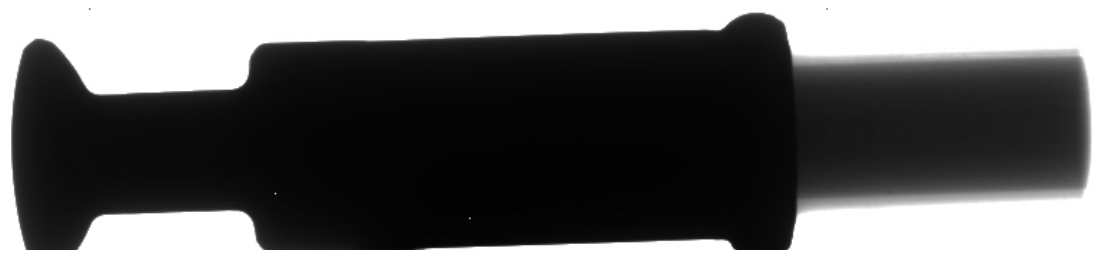

Figure $5 \mathrm{DR}$ detection at the end of composite insulator $(55 \mathrm{kV}, 3 \mathrm{~mA})$ 


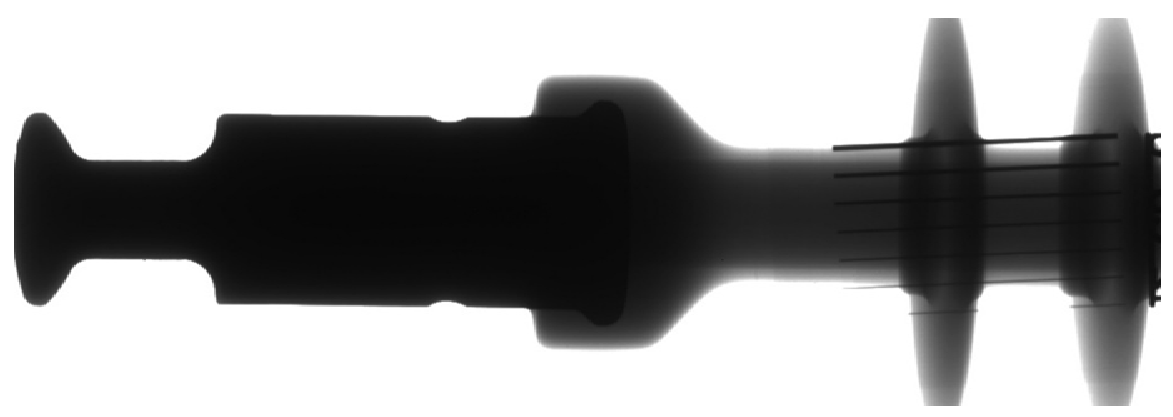

Figure 6 DR detection at the end of composite insulator $(60 \mathrm{kV}, 2 \mathrm{~mA})$
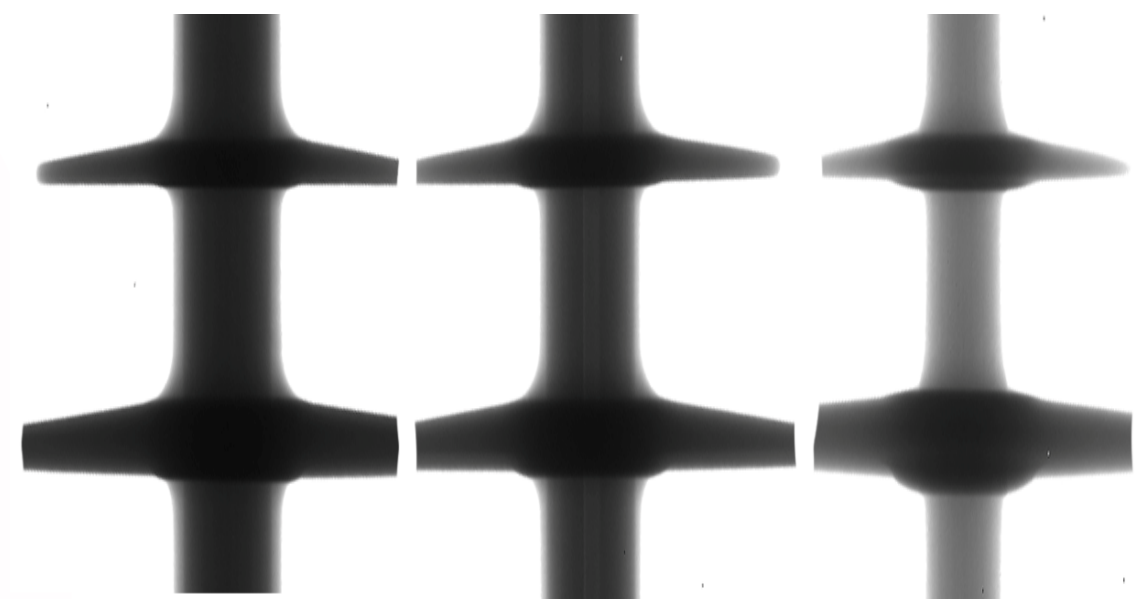

Figure 7 The imaging thickness of the insulator sheath under different transillumination parameters (a: $60 \mathrm{kV}, 2.0 \mathrm{~mA}$; b: $65 \mathrm{kV}, 2.0$ $\mathrm{mA}$; $\mathrm{c}: 65 \mathrm{kV}, 2.5 \mathrm{~mA}$ )

\section{4 conclusion}

(1) By customizing the square test block, the sound velocity of the sound wave in the composite insulator sheath rubber can be accurately measured, which lays the foundation for measuring the thickness of the composite insulator sheath. The phased array detection technology can accurately detect the thickness of the sheath, so that defects such as uneven thickness of the sheath (decentration of the core rod) can be detected, and the thickness of the sheath at the end of the composite insulator can also be effectively detected.

(2) The DR detection technology can effectively identify the interface between the mandrel and the sheath, but there is a risk of the sheath thickness distortion due to inappropriate transillumination parameters.

\section{Reference}

1. Liang Xidong, Dai Jianjun, Zhou Yuanxiang, Chen Yifang, Ultrasonic method for detecting brittle fracture cracks of FRP mandrel for insulators, Proceedings of the Chinese Society for Electrical Engineering, DOI: $\quad 10.13334 / \mathrm{j} .0258$ 8013.pcsee.2005.03.021

2. Xie Congzhen, He Zilan, Ling Yongxing, Li Lixi, Zhang Fuzeng, Li Ruihai, Phased array ultrasonic detection of internal defects in composite insulators, Proceedings of the Chinese Society of Electrical Engineering, DOI: $10.13334 / \mathrm{j} .0258$ - 8013.pcsee.2012.s1.038

3. Xie Congzhen, Zhang Yao, Hao Yanpeng, Yuan Xuejun, Wei Qianghua, Using ultrasonic flaw detector to detect internal defects of composite insulators, high voltage technology, DOI: 10.13336/j.1003-6520.hve.2009.10.011

4. Hui Qiang, Wang Gang, Application of Acoustic Emission Technology in the Crimping of Composite Insulator End Fittings, China Electric Power Education, 2010, 325-328

5. Sun Wenjian, Liu Xuandong, Deng Tao, Zhou Jun, Zhang Qiaogen, Research status of typical defect detection methods for composite insulator core rods, Shaanxi Electric Power, 2016 (44) 52-56

6. Li Jicheng, Kong Lingchang, Li Huan, Application of X-ray digital imaging technology in defect diagnosis of UHV basin insulators, non-destructive testing, 2018 (42) 22-24

7. Yan Wenbin, Wang Dada, Li Weiguo, Zhao Xianping, Yu Hong, Peng Qingjun, Xu Zhuo, Zhang Hui, X-ray transillumination detection and diagnosis of internal defects in composite insulators, High Voltage Electrical Apparatus, 2012 (48) 58-64

8. Yin Kuilong, Chen Cong, Sun Xuewu, Li Zhengli, Feng Yunguo, Application research of industrial $\mathrm{CT} / \mathrm{DR}$ detection system in basin insulator detection, Shandong Electric Power Technology, 2018 (45) 3336 